Support Information

\title{
The Role of Calcium in Regulating the Conformational Dynamics of D-Galactose/D-Glucose-Binding Protein Revealed by Markov State Model Analysis
}

Maohua Yang ${ }^{l, \#}$, Yegen Tang ${ }^{l, ~ \#, ~ J i n g w e i ~ W e n g ~}{ }^{l,}{ }^{*}$, Zhijun Liu ${ }^{2},{ }^{*}$, Wenning Wang ${ }^{l,}$ *

${ }^{1}$ Department of Chemistry, Multiscale Research Institute of Complex Systems and Institute of Biomedical Sciences, Fudan University, Shanghai 200438, China

${ }^{2}$ National Facility for Protein Science in Shanghai, Zhangjiang Lab, Shanghai

Advanced Research Institute, Chinese Academy of Sciences, Shanghai 201210, China

\# these authors contribute equally to the work

* Corresponding authors

Jingwei Weng: jwweng@,fudan.edu.cn

Zhijun Liu: liuzhijun@sari.ac.cn

Wenning Wang: wnwang@fudan.edu.cn 


\section{Method}

\section{Calculation of the bending and twisting angle}

The angle between the intersecting lines connecting the center of mass of the hinge region and those of the $\mathrm{C}$-terminal domain and $\mathrm{N}$-terminal domain is defined as bending angle, while the twisting angle formed by the centers of mass of the $\mathrm{N}$-terminal domain, the $\mathrm{N}$-terminal part of hinge region, the $\mathrm{C}$-terminal part of hinge region and the $\mathrm{C}$-terminal domain defines the twisting angle. ${ }^{1}$

\section{Calculation of generalized correlation coefficients}

Generalized correlations coefficients for the residues in the GGBP were calculated based on mutual information between all $\mathrm{C}_{\alpha}$ atoms in the protein using the generalized correlation analysis approach. ${ }^{2}$ The g_correlation module in the GROMACS package ${ }^{3}$ was applied for the analysis.

\section{NMR experiments}

All NMR experiments were carried out at $25{ }^{\circ} \mathrm{C}$ on Bruker Avance III HD $900 \mathrm{MHz}$ spectrometer equipped with cryogenic probe. ${ }^{1} \mathrm{H}-{ }^{15} \mathrm{~N}$ HSQC spectra of apo-GGBP and GGBP saturated with $\mathrm{Ca}^{2+}$ (1:20 molar ratio) were recorded respectively. NMR spectra were processed with the NMRpipe software package ${ }^{4}$ and analyzed with PIPP $^{5}$ and Sparky. ${ }^{6}$ 


\section{Isothermal calorimetric titration}

Isothermal calorimetric titration (ITC) measurements were performed on an MicroCal PEAQ-ITC calorimeter (Malvern Instruments Ltd, United Kingdom) at $20{ }^{\circ} \mathrm{C}$. All protein samples were dissolved in a buffer containing $20 \mathrm{mM}$ Tris, $\mathrm{pH} 8.0,100 \mathrm{mM}$ $\mathrm{NaCl}$. The titrations were carried out by injecting $40-\mu \mathrm{l}$ aliquots of apo-GGBP or GGBP/Ca ${ }^{2+}(0.47 \mathrm{mM})$ into glucose $(0.05 \mathrm{mM})$ at time intervals of 2 min to ensure that the titration peak returned to the baseline. ITC data was analyzed using the Malvern MicroCal PEAQ ITC software (Malvern Instruments Ltd) and fitted by the the Wiseman isotherm binding model. ${ }^{7-9}$

\section{Markov state model construction}

To validate our model, we also used the $\mathrm{IICA}^{10}, 11$ method combined with K-Centers, ${ }^{12,}{ }^{13}$ Mini Batch K-Medoids, ${ }^{14}$ and Mini Batch K-means algorithms ${ }^{15}$ to divide the conformational space. The backbone dihedral angles $(\Phi, \Psi)$ of 305 residues are used as the feature for tICA. The cosine and sine values of all the dihedral angles were also calculated and used together with the dihedral angles as features for every structure (totally $305 \times 4=1220$ ). The tICA method was adopted to reduce the 1220-dimension and capture the slow motions. Optimal parameters for tICA reduction and MSM construction were selected using a generalized matrix Rayleigh quotient $(\mathrm{GMRQ})^{10,16}$ and parameters including tICA number, tICA lag time, cluster method and the number of clusters (Figure S3). Here, we performed 5 folds of cross validation by randomly selecting $80 \%$ number of trajectories as the training set for learning 
MSM and used the remaining trajectories as test for scoring. According to the GMRQ scores in Figure S3, K-Centers and Mini Batch K-Medoids algorithms give the optimal number of states 50 for both apo-GGBP and GGBP/Ca ${ }^{2+}$ systems. Therefore, MSMs with 50 states were constructed and the 2D-PMFs were calculated from the MSMs (Figure S4).

\section{Classification of macro-sates and mean first passage time calculation}

To calculate the mean first passage time (MFPT), we lumped microstates into two macro-states according to,

$$
\begin{gathered}
f\left(S_{i}\right)= \\
\left\{\begin{array}{r}
\text { closed state, } 18 * x_{i}^{\text {bending }}-2376-y_{i}^{\text {twisting }}<0 \\
\text { open state }, 18 * x_{i}^{\text {bending }}-2376-y_{i}^{\text {twisting }} \geq 0
\end{array}\right.
\end{gathered}
$$

where $S_{i}$ is the center of every micro-states. ( $x_{i}^{\text {bending }}, y_{i}^{\text {twisting }}$ ) is the bending and twisting angle value of the center of the micro-state $S_{i}$.

Then, we followed the procedure in $\operatorname{Ref}^{17}$ to compute the mean first passage time (MFPT) from initial state $i$ to final state $f$, i.e. the average time taken to get from state $i$ to state $f$ for the first time.

$$
M F P T_{i f}=\sum_{j} P_{i j}\left(\tau+M F P T_{j f}\right)
$$


where $P_{i j}$ is the transition probability from state $i$ to state $j, \tau$ is the lag time of the transition probability matrix $\boldsymbol{T}$, and $M F P T_{j f}$ is the mean first passage time of the state $j$ to final state $f$. The boundary condition is $M F P T_{f f}=0$. 

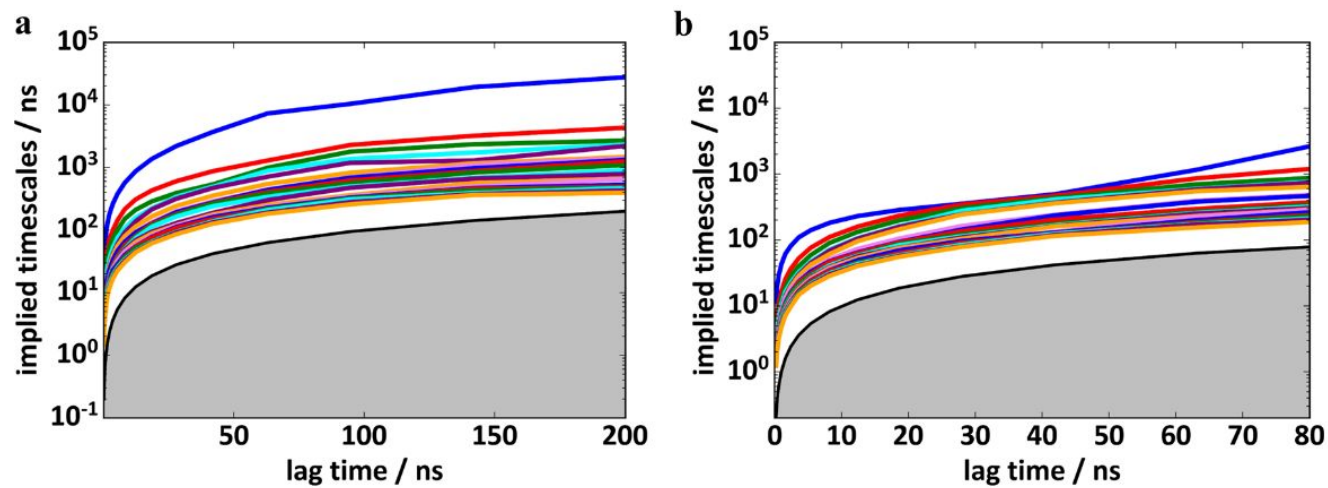

Figure S1. The variations of implied timescales with lag time for apo-GGBP (a) and GGBP/Ca ${ }^{2+}(b)$. 

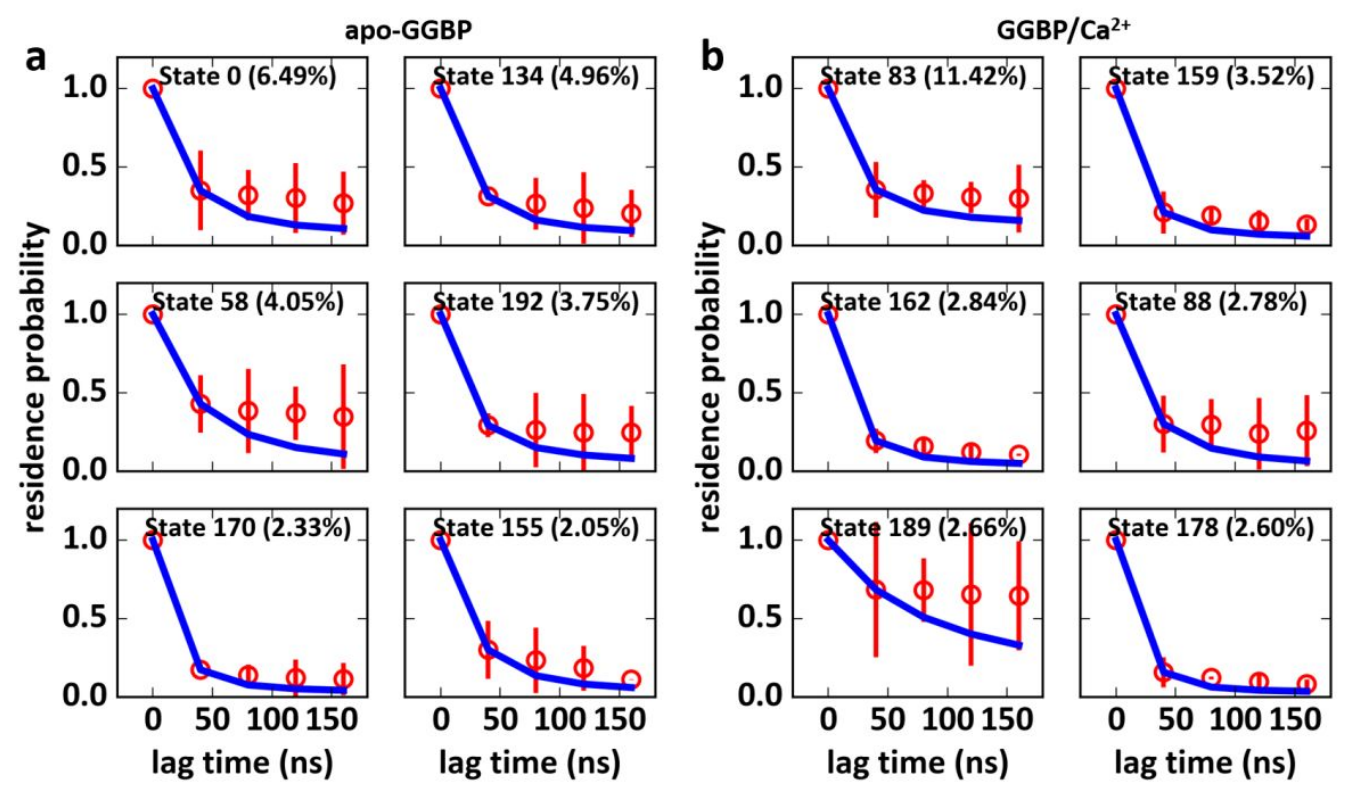

Figure S2. The Chapman-Kolmogorov tests of top six population microstates for apo-GGBP (a) and GGBP/Ca ${ }^{2+}(b)$. The error analyses were performed for the test using the boostrap method. 

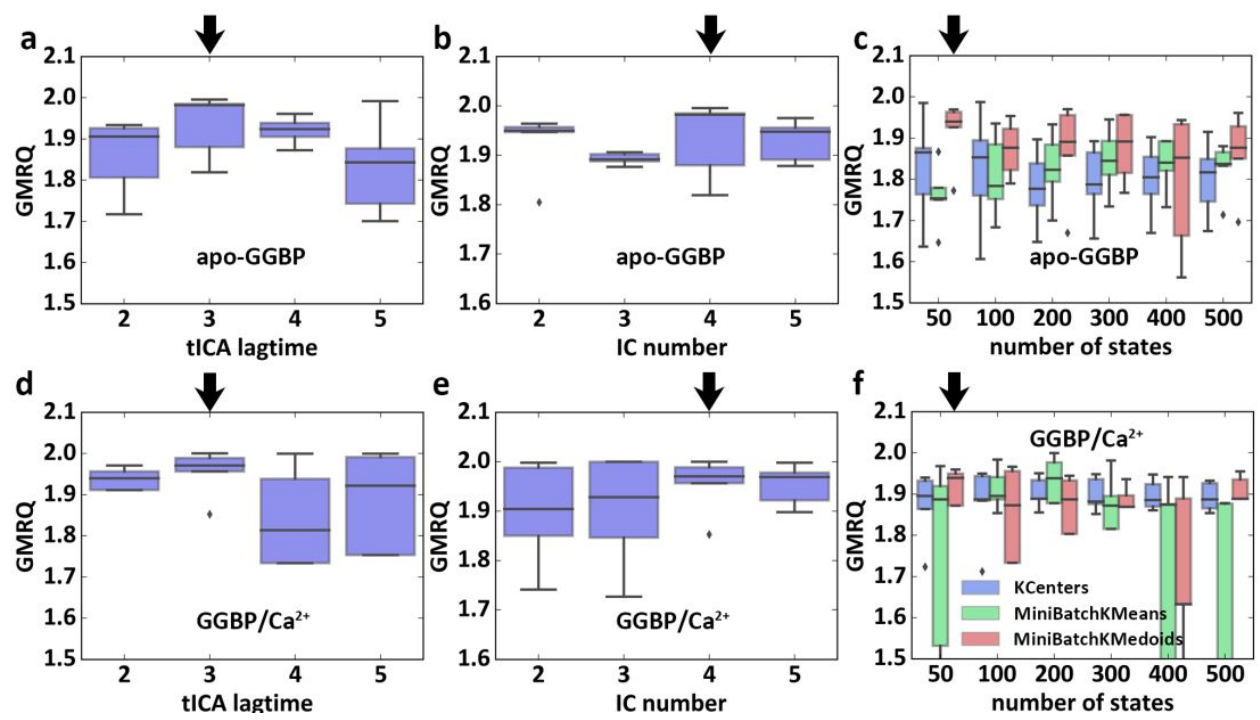

Figure S3. Box plot for GMRQ tests of the parameters for MSM construction, including tICA lag time, IC number and state number. The optimal parameters are denoted with the black arrows (a-c for apo-GGBP and d-f for GGBP/Ca ${ }^{2+}$ ). 

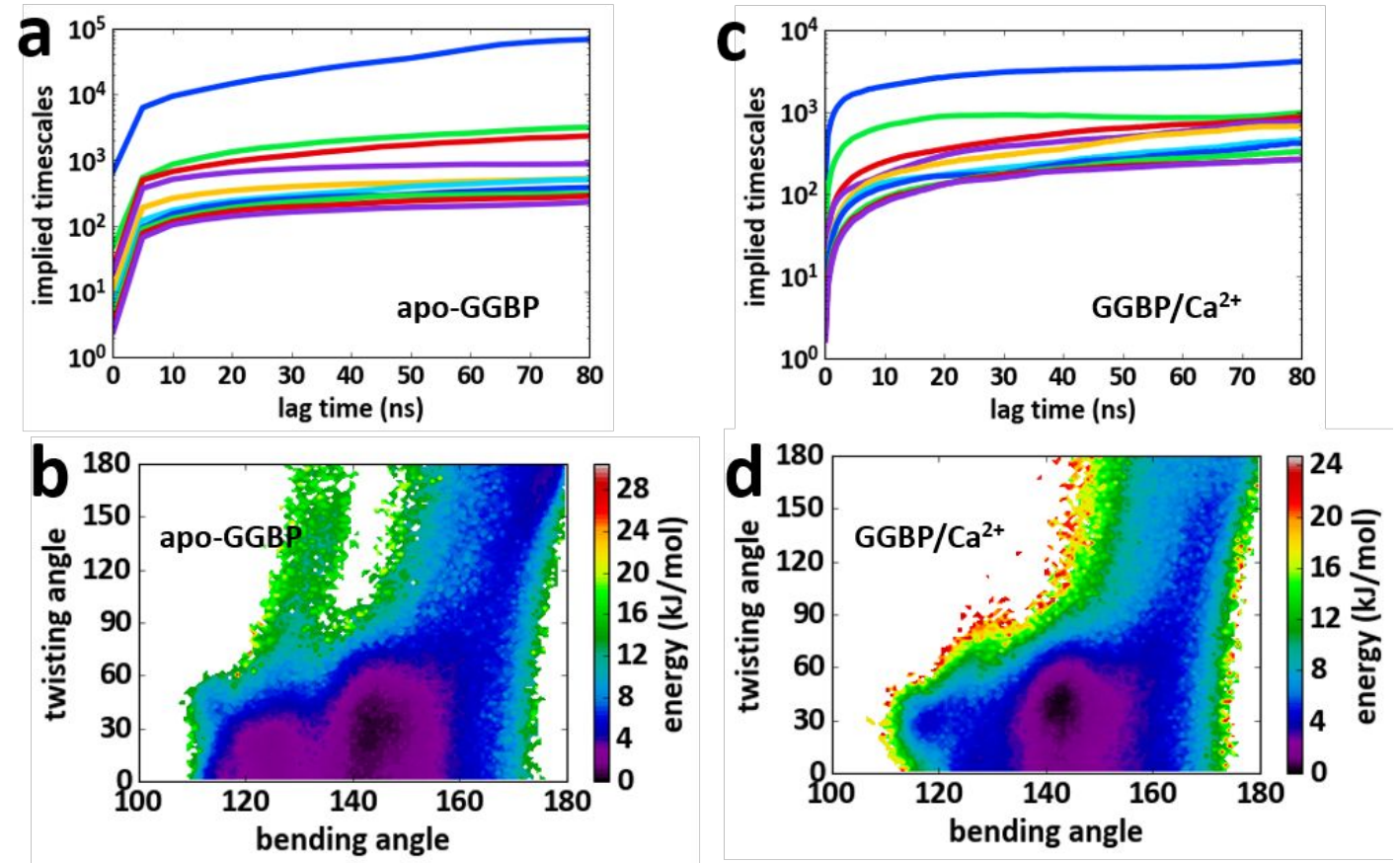

Figure S4. Implied timescale and the calculated free energy landscape of the 50-state MSM constructed by using tICA method for apo-GGBP a-b and GGBP/Ca ${ }^{2+} \mathrm{c}-\mathrm{d}$. 


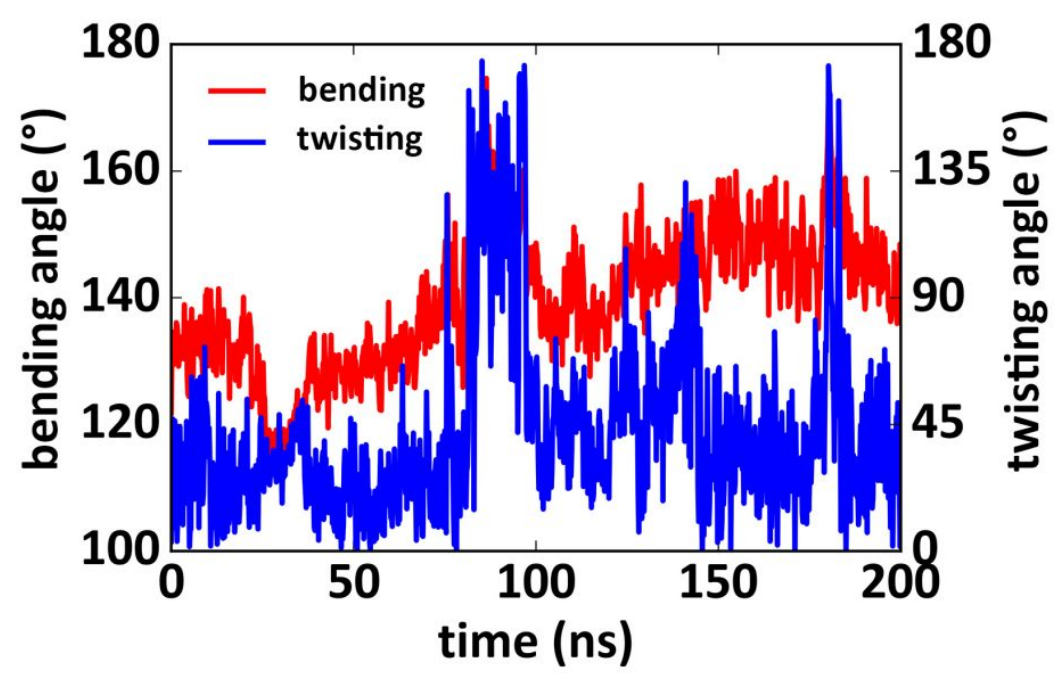

Figure S5. The time variations of the bending (red) and twisting (blue) angles along a selected trajectory. The values of the two angles fluctuate wildly (bending angle: $120^{\circ} \sim 180^{\circ}$, twisting angle: $0^{\circ} \sim 180^{\circ}$ ). 


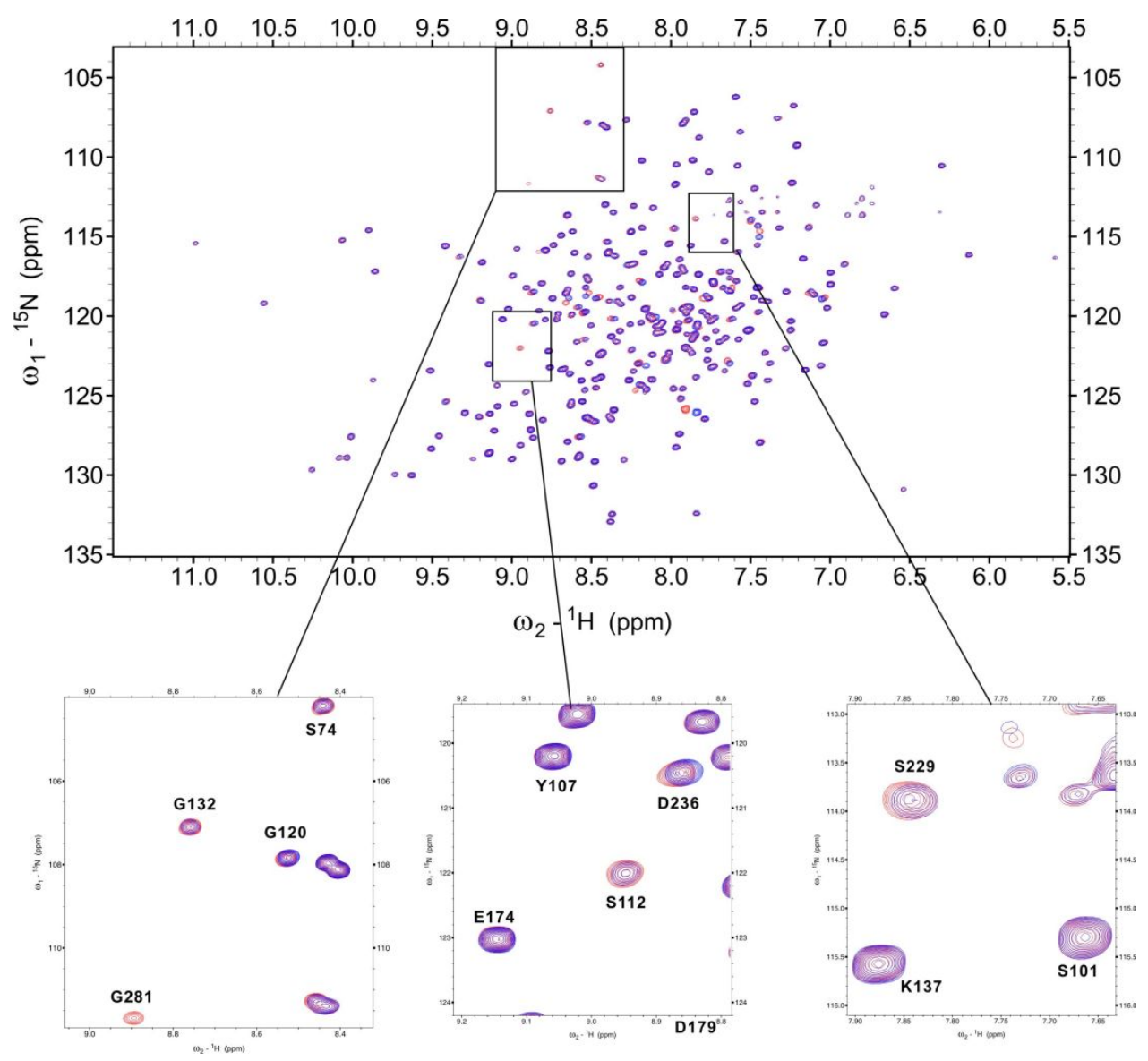

Figure S6. The ${ }^{1} \mathrm{H}^{15} \mathrm{~N}$ HSQC spectra of apo-GGBP (blue) and GGBP/Ca ${ }^{2+}$ (red) with the closed-up views of some areas. 


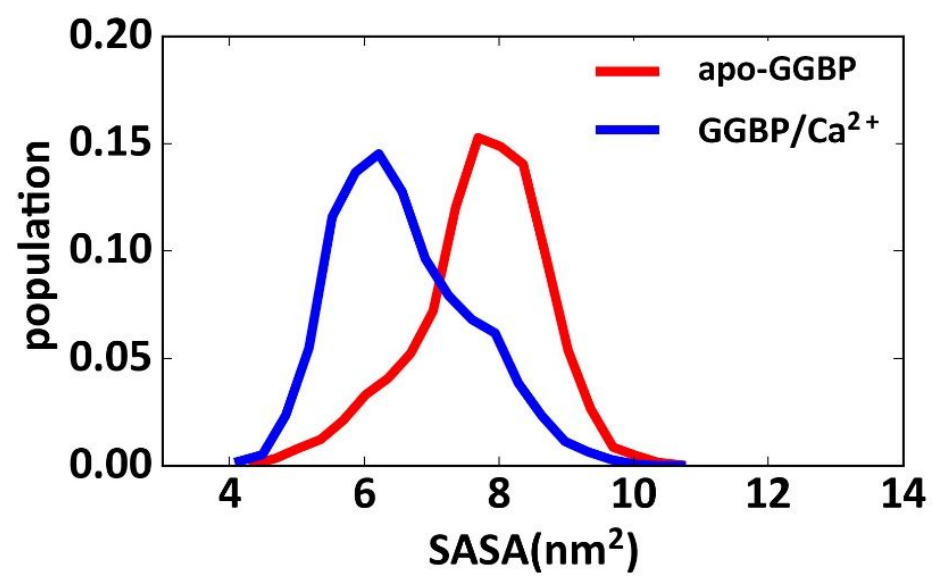

Figure S7. SASA distributions of the $\mathrm{Ca}^{2+}$ binding pocket in apo-GGBP (red) and $\mathrm{GGBP} / \mathrm{Ca}^{2+}$ (blue). 

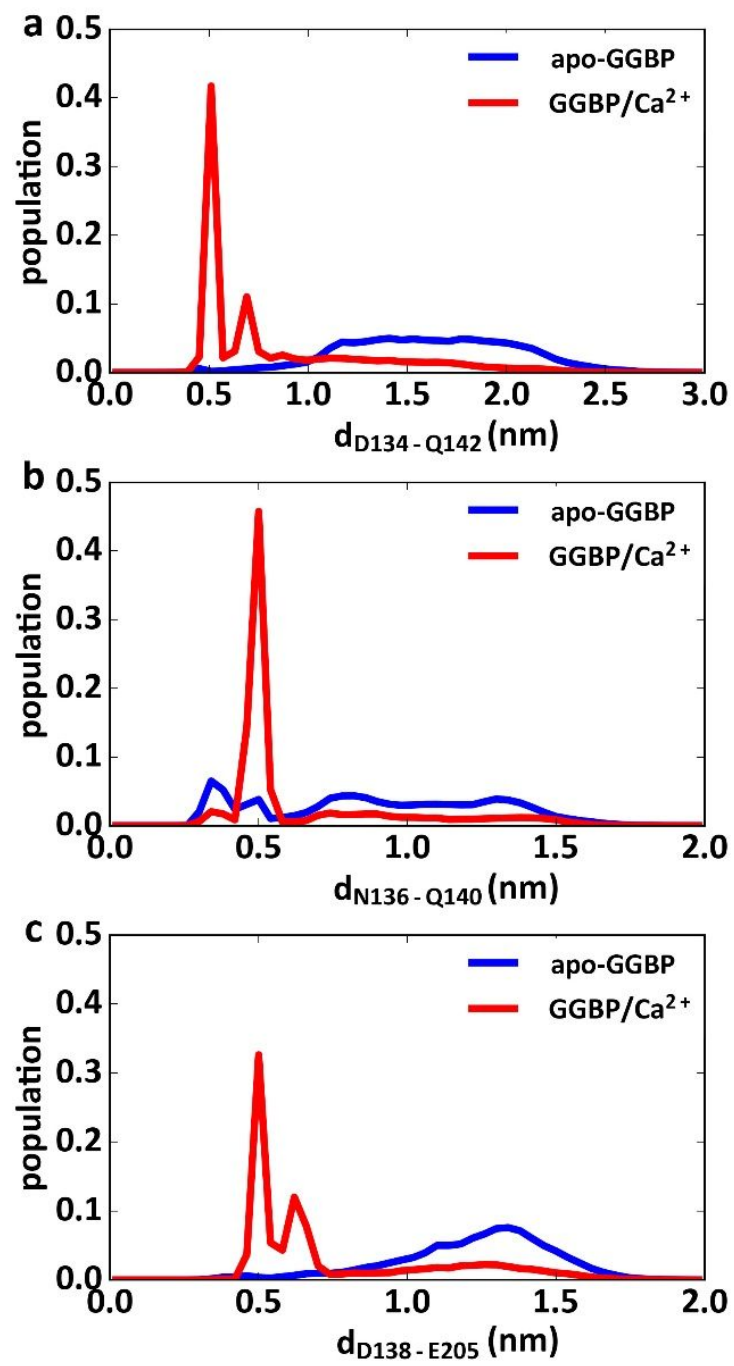

Figure S8. Distance distributions of three atom pairs inside the $\mathrm{Ca}^{2+}$-binding pocket of apo-GGBP (blue) and $\mathrm{GGBP} / \mathrm{Ca}^{2+}$ (red) systems. The atom pairs are: Asp134-OD1:Gln142-OE1, Asn136-OD1:Gln140-O and Asp138-OD2:Glu205-OE1. 


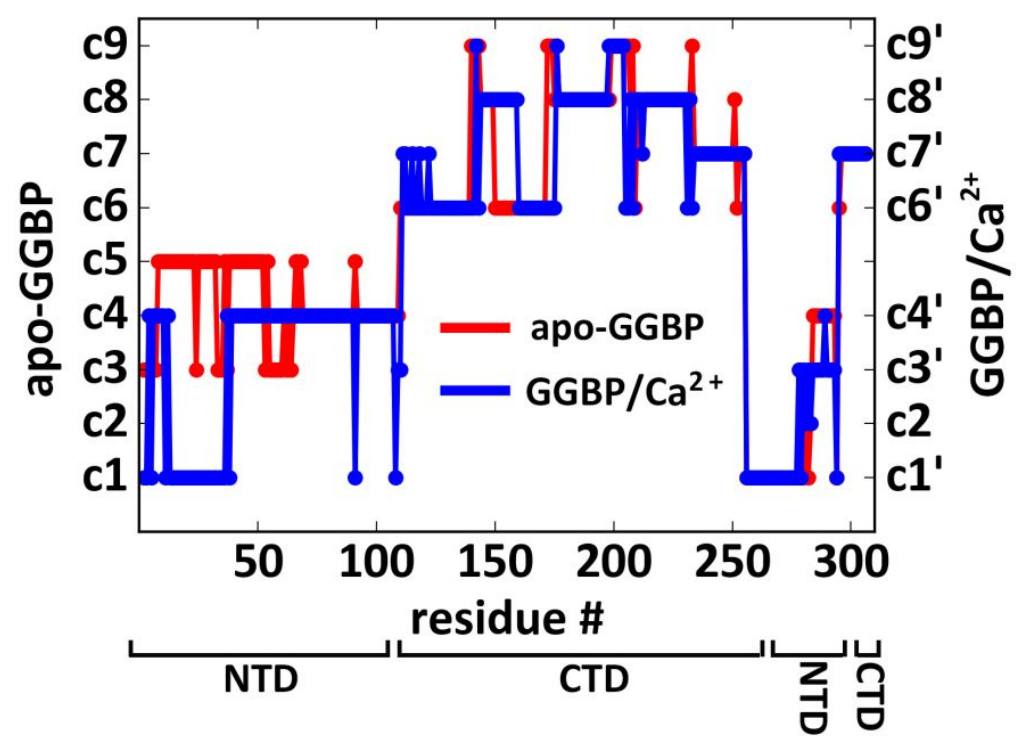

Figure S9. The assignments of communities for all the residues in apo-GGBP (blue) and $\mathrm{GGBP} / \mathrm{Ca}^{2+}($ red) systems. 


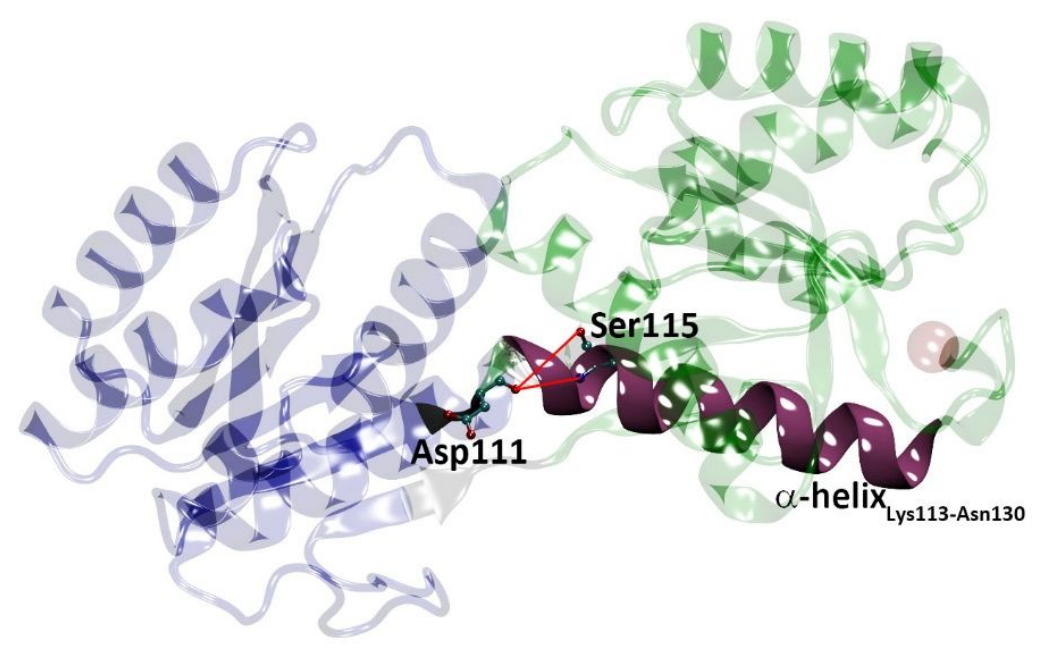

Figure S10. Hydrogen bonds between Ser115 and Asp111. The first $\alpha$-helix (Lys113-Asn130) of C-terminal domain is colored in mauve. 

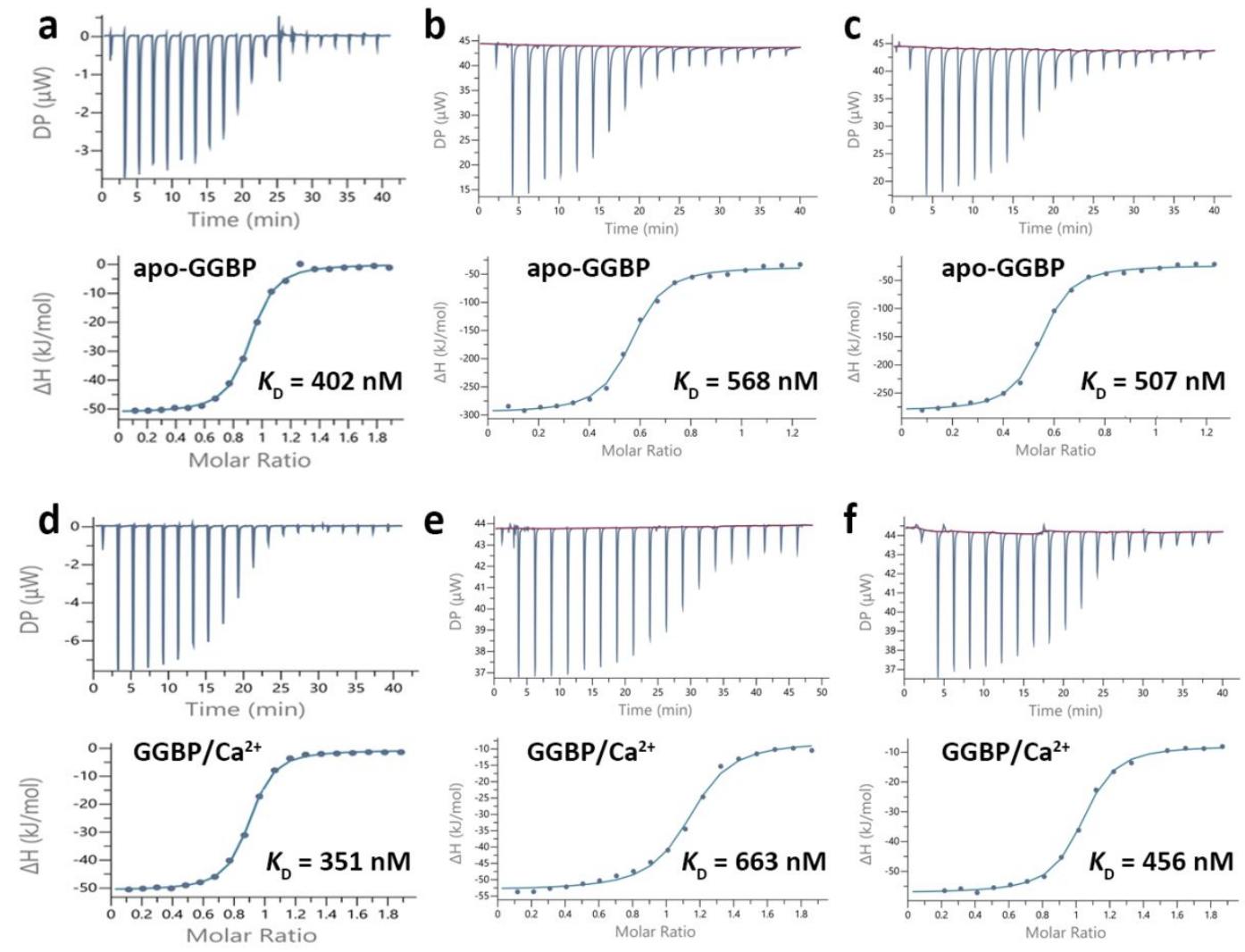

Figure S11. Isothermal calorimetric titration (ITC) experiments that measure the glucose binding affinities of apo-GGBP (a-c) and GGBP/Ca ${ }^{2+}(d-f)$. 

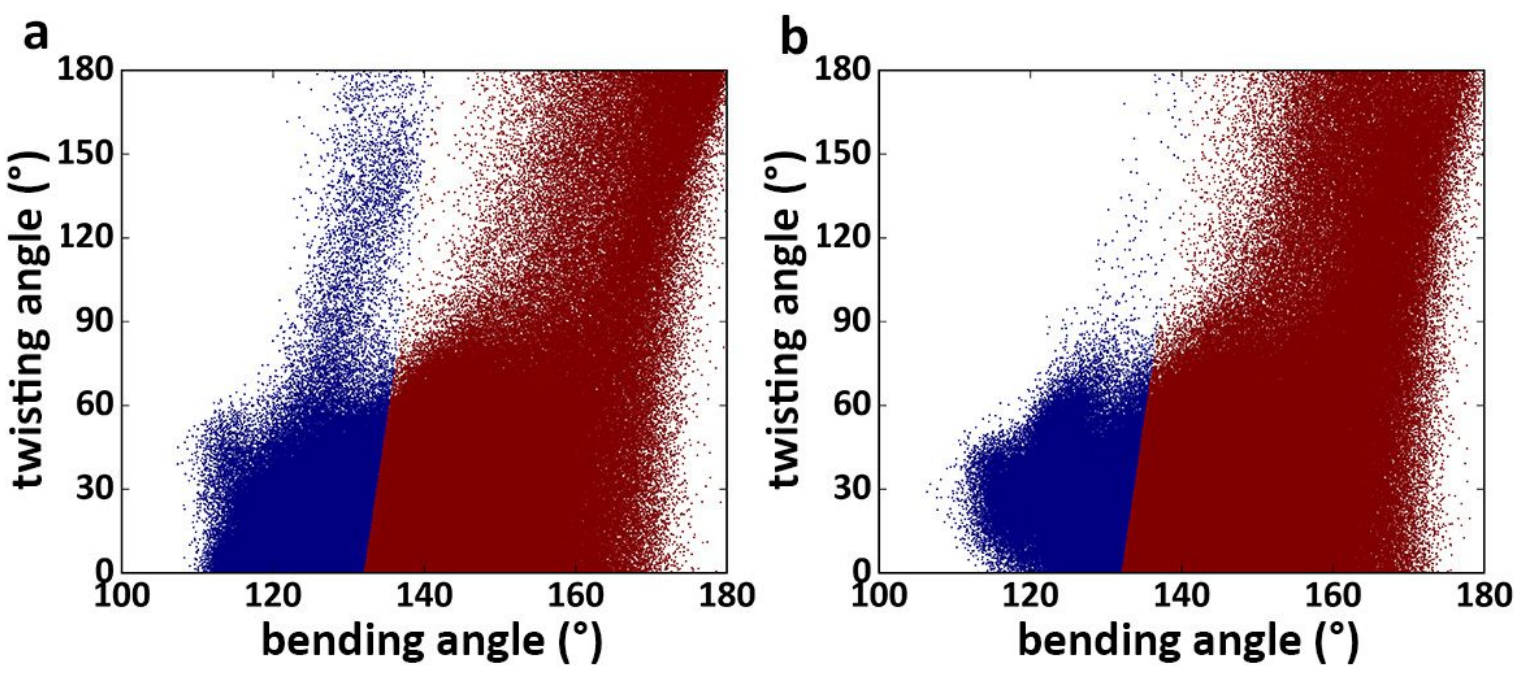

Figure S12. The two-state MSM for apo-GGBP (a) and for GGBP/Ca ${ }^{2+}$ (b). 


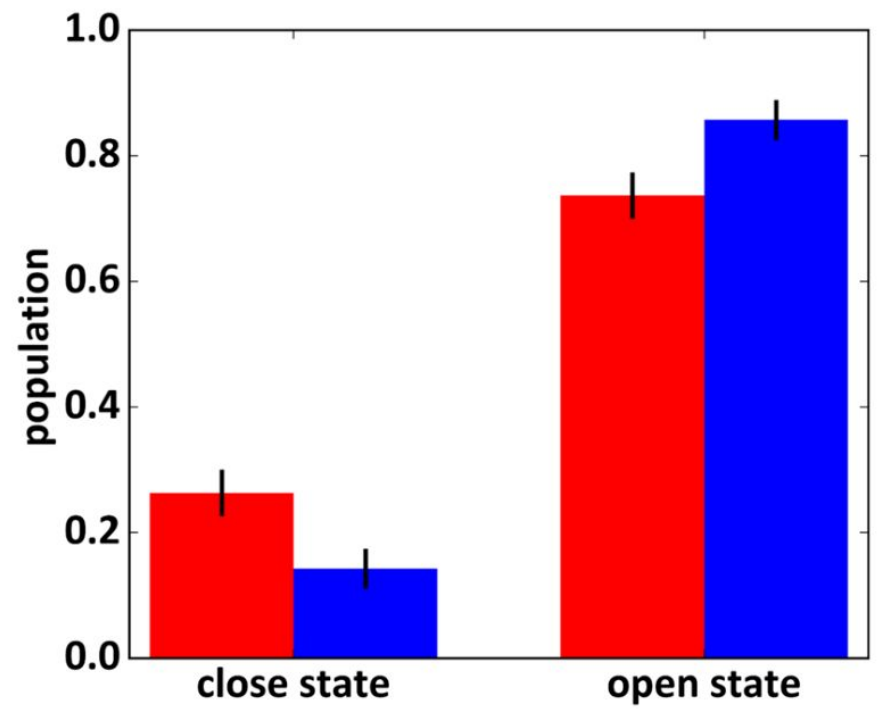

Figure S13. Populations of the closed and open states in the two-state MSM for apo-GGBP (red) and GGBP/Ca ${ }^{2+}$ (blue).

Table S1. Details of the simulation systems

\begin{tabular}{ccccc}
$\begin{array}{c}\text { Simulation } \\
\text { system }\end{array}$ & \#ions & \#water & \#atom & Simulation time $(\mu \mathrm{s})$ \\
\hline apo-GGBP & $0.1 \mathrm{M}\left(46 \mathrm{Na}^{+} 38 \mathrm{Cl}^{-}\right)$ & 23045 & 73851 & 49.34 \\
& & & & \\
$\mathrm{GGBP} / \mathrm{Ca}^{2+}$ & $0.1 \mathrm{M}\left(44 \mathrm{Na}^{+} 38 \mathrm{Cl}^{-} 1 \mathrm{Ca}^{2+}\right)$ & 23045 & 73850 & 48.82 \\
& & & & \\
\hline
\end{tabular}


Table S2. The variations of hydrogen bonds upon $\mathrm{Ca}^{2+}$-binding.

Frequency* $(\%)$

\begin{tabular}{|c|c|c|c|c|}
\hline \multirow[t]{2}{*}{ Donor } & \multirow[t]{2}{*}{ Acceptor } & \multirow[b]{2}{*}{ apo-GGBP } & \multirow[b]{2}{*}{ GGBP/Ca ${ }^{2+}$} & \multirow[b]{2}{*}{$\Delta^{* *}$} \\
\hline & & & & \\
\hline Gln142-NE2 & Asp138-OD1 & 1.6771 & 39.7751 & 38.098 \\
\hline Gln142-N & Glu205-OE1 & 12.5377 & 32.8018 & 20.2641 \\
\hline Gln142-NE2 & Asp138-OD2 & 1.7526 & 24.3407 & 22.588 \\
\hline Lys137-N & Asp134-OD1 & 10.9122 & 37.2498 & 26.3376 \\
\hline $\mathrm{G} \ln 142-\mathrm{N}$ & Glu205-OE2 & 11.3963 & 33.8059 & 22.4095 \\
\hline Gln142-NE2 & Glu174-OE2 & 11.3403 & 31.5245 & 20.1842 \\
\hline Lys137-N & Asp134-OD2 & 10.5792 & 31.6666 & 21.0874 \\
\hline Gln140-NE2 & Asp138-OD1 & 5.9991 & 34.2535 & 28.2544 \\
\hline Gln $140-\mathrm{N}$ & Asp138-OD2 & 1.2704 & 30.5177 & 29.2473 \\
\hline Gln142-NE2 & Glu174-OE1 & 9.9975 & 32.6351 & 22.6376 \\
\hline Leu135-N & Glu205-OE2 & 5.0085 & 27.3315 & 22.3229 \\
\hline Ser115-OG & Asp111-O & 35.2002 & 14.9931 & -20.2071 \\
\hline Ala237-N & Val254-O & 57.7135 & 37.2926 & -20.4208 \\
\hline
\end{tabular}




$\begin{array}{lllll}\text { Ser115-N } & \text { Asp111-O } & 41.4467 & 20.1248 & -21.3218\end{array}$

*Here we list the hydrogen bonds which $|\Delta|>=0$.

$* * \Delta=$ Frequency $\left(\mathrm{GGBP} / \mathrm{Ca}^{2+}\right)-$ Frequency(apo-GGBP).

\section{Reference}

1. Unione, L.; Ortega, G.; Mallagaray, A.; Corzana, F.; Pérez-Castells, J.; Canales, A.;

Jiménez-Barbero, J.; Millet, O., Unraveling the conformational landscape of ligand binding to glucose/galactose-binding protein by paramagnetic nmr and md simulations. ACS chem. Biol. 2016, 11, 2149-2157.

2. Lange, O. F.; Grubmüller, H., Generalized correlation for biomolecular dynamics.

Proteins Struct. Funct. Bioinformat. 2006, 62, 1053-1061.

3. Hess, B.; Kutzner, C.; Van Der Spoel, D.; Lindahl, E., Gromacs 4: Algorithms for highly efficient, load-balanced, and scalable molecular simulation. J. Chem. Theory Comput. 2008, 4, 435-447. 
4. Delaglio, F.; Grzesiek, S.; Vuister, G. W.; Zhu, G.; Pfeifer, J.; Bax, A., Nmrpipe: A multidimensional spectral processing system based on unix pipes. J. Biomol. NMR 1995, 6, 277-293.

5. Garrett, D. S.; Powers, R.; Gronenborn, A. M.; Clore, G. M., A common sense approach to peak picking in two-, three-, and four-dimensional spectra using automatic computer analysis of contour diagrams. J. Magn. Reson. 2011, 213, 357-363.

6. Lee, W.; Tonelli, M.; Markley, J. L., Nmrfam-sparky: Enhanced software for biomolecular nmr spectroscopy. Bioinformatics 2015, 31, 1325-1327.

7. Wiseman, T.; Williston, S.; Brandts, J. F.; Lin, L.-N., Rapid measurement of binding constants and heats of binding using a new titration calorimeter. Anal. Biochem. 1989, 179, 131-137.

8. Freire, E.; Mayorga, O. L.; Straume, M., Isothermal titration calorimetry. Anal. Chem. 1990, 62, 950A-959A.

9. Brown, A., Analysis of cooperativity by isothermal titration calorimetry. Int. J. Mol. Sci. 2009, 10, 3457-3477.

10. Pérez-Hernández, G.; Paul, F.; Giorgino, T.; De Fabritiis, G.; Noé, F., Identification of slow molecular order parameters for markov model construction. J. Chem. Phys. 2013, 139, 07B604_601.

11. Schwantes, C. R.; Pande, V. S., Improvements in markov state model construction reveal many non-native interactions in the folding of nt19. J. Chem. Theory Comput. 2013, 9, 2000-2009. 
12. Gonzalez, T. F., Clustering to minimize the maximum intercluster distance. Theor.

Comput. Sci. 1985, 38, 293-306.

13. Beauchamp, K. A.; Bowman, G. R.; Lane, T. J.; Maibaum, L.; Haque, I. S.; Pande, V. S., Msmbuilder2: Modeling conformational dynamics on the picosecond to millisecond scale. J. Chem. Theory Comput. 2011, 7, 3412-3419.

14. de Hoon, M. J. L.; Imoto, S.; Nolan, J.; Miyano, S., Open source clustering software. Bioinformatics 2004, 20, 1453-1454.

15. Sculley, D., In Proceedings of the 19th international conference on World wide web; Association for Computing Machinery: Raleigh, North Carolina, USA, 2010, pp 1177-1178.

16. McGibbon, R. T.; Pande, V. S., Variational cross-validation of slow dynamical modes in molecular kinetics. J. Chem. Phys. 2015, 142, 03B621_621.

17. Singhal, N.; Snow, C. D.; Pande, V. S., Using path sampling to build better markovian state models: Predicting the folding rate and mechanism of a tryptophan zipper beta hairpin. J. Chem. Phys. 2004, 121, 415-425. 